\title{
The role of the practice nurse in the management of asthm
}

\author{
Rupert C M Jones, Sam Freegard, Mark Reeves, Karen Hanney, Frank Dobb
}

\author{
TBSTRAC \\ Backgroun \\ The role of the practice nurse may include diagnosis an \\ management of asthma, this study examines the range of activitie \\ performed by nurses and their training
}

\section{Aims}

To ascertain the role and confidence levels of the practice nurse i diagnosis and management of asthmatic patients

\author{
Method \\ 9 postal questionnaire sent to the named respiratory nurse in 17 \\ practices in Cornwall and Southwest Devon, to assess the number o \\ practice nurses offering asthma management, extent of services an \\ confidence level of nurses in this role
}

\section{Results}

The response rate was 64\%: Dedicated asthma clinics operated i $47 \%$ of practices, $87 \%$ undertaken by the nurse alone

$\mathbb{R}$ esponsibilities undertaken by nurses alone included: instruction o inhaler technique $93 \%$, supervising self-management plans $87 \%$ ,changing medication dosage $71 \%$, withdrawing treatment $53 \%$ diagnosing asthma $45 \%$ and managing acute exacerbations $29 \%$ Nurses initiated treatment alone, without consulting a doctor, a follows; inhaled bronchodilators 55\%, long acting bronchodilator $54 \%$, inhaled steroids $56 \%$, oral steroids $15 \%$, anti-leukotrienes 5 gnd theophyllines $3 \%$. The confidence level of the nurses performin these tasks was high. Formal training had been undertaken by 74 of respondents. There were statistically significant association between performance of organisational tasks and training, bu durprisingly no apparent statistical associations with training an independent initiation of treatments.

\section{Conclusion}

Practice nurses are performing activities previously undertaken $b$ goctors. A minority have not had formal training and performin these activities, without well-defined shared care protocols, may b outside current legal frameworks

\section{Keywords}

Practice nurses, Asthma, Primary care, Questionnaire

\section{Rupert C M Jone \\ General Practitione \\ Introductio}

\section{Bam Freegar}

General Practitione

\section{Mark Reeve}

General Practitioner,

\section{Karen Hanney \\ NG}

\section{Frank Dobb}

Senior Lecture

Correspondence to

Đr Rupert C M Jone

$\mathbb{R}$ espiratory Researc

Uni

Department of Primar

Health Care \& Genera

Practic

gTTC Buildin

Kamar Science Par

$\mathbb{1}$ Davy Roa

Derrifor

Plymout

trupertjones@ukgateway.ne

Date Submitted: $14 / 11 / 0$

Date Accepted: 20/11/0

Prim Care Respir 2001 10(4) $109-11$ isolated.

\section{Method}

Subject
There is little doubt that the prevalence of asthma $\mathrm{i}$ growing nationally and globally ${ }^{3-}$ There is increasin fvorkload in primary care, both in the number o people consulting for asthma for the first time and $\mathrm{i}$ total asthma consultations ${ }^{4}$ While recent evidence ha shown that GP consultations for asthma may hav peaked in the 1990's ${ }^{5}$ this did not include practic nurse consultations. Nurses are now taking a majo role in asthma management in primary care 6

Duidelines for the management of asthma and COP have been issued ${ }^{8}$, but do not clarify the roles o doctors and nurses. What is not known is whethe nurses have received adequate training and support fo their extending roles. Doctors, in passing work t gurses, may become de-skilled, further reducin support for their practice nurses. There is a risk tha primary care nurses may become professionall

This study seeks to assess the role and responsibilitie of the practice nurse in caring for patients with asthm in general practice in the South West

The practice nurse with prime responsibility fo ssthma management was identified by telephone call to the receptionist or practice manager in ever practice in the South West Devon Health Authorit area, (including Plymouth and Torbay), and in th Cornwall and Isles of Scilly Health Authority area
This named individual (one per practice) received postal questionnaire.

\section{The questionnair}

The questionnaire included sections on responsibilitie dnd activities of the nurse in asthma, COPD an epirometry. The questionnaire was produced by th project management team in conjunction with fou yonsultants in respiratory medicine. It was piloted $b$ groups of practice nurses outside the study area o two occasions.

It included questions on existing asthma management \$sthma clinics, the responsibilities the nurse nndertook alone and their confidence levels i gerforming those duties. Formal asthma trainin details were requested, further questions abou dpirometry and COPD management are reporte separately (pages 106-8). One hundred and sevent oeven nurses were sent questionnaires. Nurses wh glid not respond in four weeks received a second cop of the questionnaire. Data was analysed using Fisher' exact test in SPSS statistical analysis software $t$ dxamine the association between the training an activities of the nurses in practice

\section{Result}

The response to the first mailing was 79 , to the secon 34 , giving a total of 113 out of 177 , a $64 \%$ respons rate.

Æn those practices holding regular clinics (47\%), th frequency of clinics ranged from four times a week $t$ 
snce per month, with the majority seeing patient nnce or twice per week. The remainder saw patients i normal clinic sessions or opportunistically. Asthm nlinic appointments were managed by nurses alone $\mathrm{i}$ $87 \%$ of the practices, joint doctor and nurse clinics i $\mathbb{t} 1 \%$, the doctor alone in $2 \%$. A frequent commen as that in those practices not using a dedicate asthma clinic, there had been a high level of non attenders in asthma clinics. They had therefor yhanged to appointments within normal surger sessions

The nurses were asked about their responsibilities i dealing with asthmatic patients alone and not $\mathrm{i}$ tconsultation with a doctor. (See Table 1). The mos frequent role was in relation to inhaler devices instructing inhaler technique (93\%), selecting (84\% find changing inhaler devices (85\%). In terms o snodifying treatment regimes, there was les fonsistency: although $87 \%$ supervised sel management plans, $71 \%$ changed doses of curren treatment and only $53 \%$ withdrew medications gwenty nine percent felt they had a role in managin kcute exacerbations. The complex and important tas of making a diagnosis was performed by $45 \%$. Detail of drug treatments initiated by nurses, alone and not $\mathrm{i}$ consultation with a doctor, are recorded in Table 2 Between 54-56\% were initiating inhaled treatments dhort and long acting beta-agonists an corticosteroids. A minority also initiated oral therapie such as steroids, theophylline and anti-leukotrien \&gents. Nurses' confidence levels for all activitie were high.

The nurses were asked if they had undergone forma asthma training i.e. leading to a diploma or othe qualification. They were asked to confirm th dualification gained. Seventy four percent reporte that they had formal training, $61 \%$ confirmed th sature of the qualification. There were many practice where more than one nurse had received training Comments were made frequently on the value of shor kourses including those organised by pharmaceutica nompanies. The statistical association betwee snitiating drug therapy by nurses and their training $\mathrm{i}$ included in Table 2

\section{Discussio}

This survey provides a balanced picture of the view dnd activities of practice nurses in Cornwall an Southwest Devon. Many previous studies involve asthma interested primary health care teams. Despite $64 \%$ response rate, there may be selection bias in tha the respondents may be more enthusiastic abou asthma management than the non-responders Furthermore, the use of multiple significance testin should be interpreted with caution

The effectiveness of practice nurses has bee demonstrated in providing respiratory care in genera practic ${ }^{9}$ and may improve patient outcomes 10,1 Patients were willing to see either doctors or nurse

\section{Table}

The number of practice nurses undertaking various responsibilities in asthm management:

yIn dealing with asthmatic patients in your practice, do you have responsibilit

filone (i.e. not in consultation with a doctor) for any of the following areas o management?

‘Please indicate your confidence in dealing with those areas of responsibility'

(11) not at all (2) not very (3) fairly (4) very confiden

\begin{tabular}{|c|c|c|c|c|}
\hline \multirow[b]{2}{*}{ Enstructing inhaler techniqu } & \multirow{2}{*}{$\begin{array}{c}\text { Numbe } \\
\text { )(Total } 113 \\
\text { イ\% } \\
105(93\end{array}$} & \multirow{2}{*}{$\begin{array}{c}\text { Mean scor } \\
\text { of nurse } \\
\text { eonfidenc } \\
9 .\end{array}$} & \multicolumn{2}{|c|}{$\begin{array}{l}\text { 1\% with forma } \\
\text { grainin } \\
\text { xp-value }\end{array}$} \\
\hline & & & 78. & $\gamma 0.004$ \\
\hline Selecting inhaler devic & $95(84$ & 8. & $\theta$ & $\gamma 0.003$ \\
\hline Changing inhaler devic & $96(85$ & 8. & 29. & $\gamma 0.013$ \\
\hline Change existing treatment dosag & $80(71$ & 3. & $\theta$ & $\mathbb{S}$ \\
\hline Withdrawing/ stopping medication & $\varnothing 0(53$ & 3. & 83. & $\gamma 0.030$ \\
\hline Supervising self management plan & $98(87$ & 3. & 69. & $\gamma 0.003$ \\
\hline Managing the recall/ review syste & $78(69$ & 8. & $\$ 2$. & $\gamma 0.009$ \\
\hline Making diagnosis of asthm & $51(45$ & 3. & 84. & $\gamma 0.032$ \\
\hline Managing acute exacerbation & $\$ 3(29$ & 3. & 84. & $\mathbb{N}$ \\
\hline
\end{tabular}

Table 2

The number of practice nurses who initiated treatments 'alone (i.e. not in consultation with a doctor)'

\begin{tabular}{|c|c|c|c|c|}
\hline \multirow[b]{2}{*}{ Initiating inhaled bronchodilator } & \multirow{2}{*}{$\begin{array}{l}\text { Numbe } \\
\gamma \% \\
\varnothing 2(55\end{array}$} & \multirow{2}{*}{$\begin{array}{c}\text { Mean scor } \\
\text { aurse confidenc } \\
3.7\end{array}$} & \multicolumn{2}{|c|}{$\begin{array}{l}\text { Ho wit } \\
\text { formal training } \\
\text { ’p-values }\end{array}$} \\
\hline & & & 8 & $\mathbb{S}$ \\
\hline Initiating inhaled corticosteroid & $63(56$ & 3,7 & 8 & $\mathbf{S}$ \\
\hline $\begin{array}{l}\text { Initiating inhaled long acting } \\
\beta \text { sagonist }\end{array}$ & $61(54$ & 3.7 & 7 & $\mathbf{S}$ \\
\hline \$nitiating oral corticosteroid & $17(15$ & 3. & 88. & $\mathbf{S}$ \\
\hline Initiating anti-leukotriene & $6(5$ & 3.7 & 8 & $\mathbf{S}$ \\
\hline \$nitiating theophyllline & B) 3 & 4 & 00 & $\mathbf{S}$ \\
\hline
\end{tabular}

about their asthma: doctors may be more accessibl and able to do home visits, but nurses may be able $\mathrm{t}$ spend longer with the patient and are often mor approachable for seemingly minor problems ${ }^{2}$

This survey demonstrates the nurses' evolving role i the diffuse and varied world of primary care. Th majority of nurses are happy to advise on inhale sechnique and do so with confidence. There is les aonfidence in more critical roles such as making diagnosis of asthma, withholding or stopping curren freatment and treating acute exacerbations. All o these decisions may carry considerable implications especially for the patients. The results show tha approximately $80 \%$ of those performing asthm patient care duties had undergone formal training Shere were statistically significant association lbetween training and performing organisationa dspects of the nurses' role; such as running clinics an yecall systems, but there were no statisticall fignificant associations with training and initiation o 
ereatments. This was surprising as there may $b$ gmportant consequences for nurses prescribin medicines independently of the GP.

dWe have demonstrated that practice nurses ar fundertaking new responsibilities in the field o prescribing. At least half felt able to initiate treatmen glone, with inhaled short and long actin bronchodilators and inhaled steroids and withou sonsultation with a GP. An unexpected finding wa that a small minority of nurses are starting patients o treatment with drugs such as theophylline and anti Lukotriene agents which are recommended by the $\mathrm{S}$ Devon and other health authorities to be initiated "b specialists only".

\$n many cases practice nurses arrange for prescription to be produced for patients without discussion with th GSP who signs the prescription. This was confirme with a number of practice nurses whom we telephone when clarifying data. There are significan implications for both the GP and the nurses $\mathrm{i}$ adopting this role. The UKCC states that nurse should undertake activities in which they ar dompetent and have appropriate training an support ${ }^{3}$ eimited prescribing is available to nurs practitioners, but no asthma medication is included $\mathrm{i}$ their current formulary ${ }^{4}$ en the future nurses may b bble to prescribe independently in area of specia expertis $\$$ anthough as in the past this would be fro a limited formulary. Currently a GP who signs prescription for a patient he has not seen, will bear th responsibility for problems that may arise from tha action, nurses would also have a share in tha responsibility ${ }^{6}$ The minority of nurses $(20 \%)$ wh have not had formal training and their delegating GP could be vulnerable to criticism from thei professional bodies and a court of law. This could als apply to nurses who have undergone formal training but not had a recent update. The widespread practic of nurse initiated prescribing should only occur if clea and agreed shared care protocols exist. In the absenc of these protocols, these actions may be impossible $t$ defend both legally and professionally

Practice nurses are providing an increasing range o services for their patients with major benefits to th patient and the health service. Their role has ofte grown in a haphazard way and serious consideratio needs to be given to the training and support fo nurses as they develop their new responsibilities

\section{stcknowledgement}

We would like to thank Dr Dave Wright, Principa \&ecturer in Medical Statistics, Department of Maths Stats, University of Plymouth and Miss Susan Parker Nursing Advisory Services, Medical Defence Unio for their expert advice in preparing this paper

\section{Editors Not}

,This paper was peer reviewed for another journal hence the very short lead time

\section{Reference}

d. Strachan DP, Butland BK, Anderson HR. Incidenc and prognosis of asthma and wheezing illness fro early childhood to age 33 in a national British cohort $B M J 1996 \mathbf{2 1}: 1195-9$

IIT xunderison HR, Butland BK, Strachan D the prevalence and severity of childhood asthma $\boldsymbol{B} M$ ;994 308 1600-4

8. Magnus P, Jaakkola JK. Secular trends in th gccurrence of asthma among children and youn hdults: critical appraisal of repeated cross sectiona surveys. BMJ $1997 \mathbf{3 1}: 1795-9$

f. Royal College of General Practitioners, Office o Population Censuses and Surveys, The Department o Health. Morbidity Statistics from General Practice Fourth National Study 1991-1992, Series MB5 No 3 London. HMSO

5. Fleming DM, Sunderland R, Cross KW, Ross AM Declining incidence of episodes of asthma: a study o trends in new episodes presenting to genera practitioners in the period 1989-98

Thora 20005 :657-61

6. Wolfe S, Price S. Delivery of asthma care: patients dse of and views on healthcare services as determine from a nationwide interview survey.

Asthma J 2000 ச :141-4

a. The British Thoracic Society, The National Asthm Campaign, The Royal College of Physicians o London et al. The British Guidelines on Asthm Management 1995 Review and Position Statement Thora 1997 \$ :S1-21.

8. British Thoracic Society. BTS guidelines for th management of chronic obstructive airways disease Thorax 1997 52(suppl 5 :S1-28

9. Lindberg M, Ahlner J, Moller M, Ekstrom T Asthma nurse practice - a resource effective approac in asthma management. Respir Med 19999 :584-8 $₫ 0$. Hoskins G, Neville RG, Smith B, Clark RG. Th sffect of a trained asthma nurse on patient outcome within general practice

Asthma in Gen Pract 1998 6:S3

al 1. Jones KP, Mullee MA. Proactive nurse run asthm care in general practice reduces asthma morbidity scientific fact or medical assumption? Br J Gen Pra 1995:497-9

H2. Paterson C, Britten N. Organising primary healt care for people with asthma: the patients perspective Br J Gen Prac 2000 o :299-303

,13. United Kingdom Central Council for Nursing Midwifery and Health Visiting. Guidelines for th administration of medicines. UKCC publications 2000 114. British Medical Association and Roya IPharmaceutical Society. London. British Nationa Formulary 1998;38

d5. Crown J. Review of prescribing, supply an fidministration of Medicines, final report. Dept o Health ; March 1999.

16. Humphries J, Green J. Nurse prescribing; 26-27 Macmillan press. Basingstoke. 1999 